\title{
Role of SUMO-1 and SUMO interacting motifs in rhesus TRIM5a-mediated restriction
}

\author{
Zana Lukic ${ }^{1 \dagger}$, Stephen P Goff ${ }^{2,3}$, Edward M Campbell ${ }^{{ }^{*}}$ and Gloria Arriagada $2,3,4^{*+}$
}

\begin{abstract}
Background: TRIM5a is a member of the tripartite motif family of proteins that restricts retroviral infection in a species-specific manner. The restriction requires an interaction between the viral capsid lattice and the B30.2/SPRY domain of TRIM5a. Previously, we determined that two SUMO interacting motifs (SIMs) present in the B30.2/SPRY domain of human TRIM5a (huTRIM5a) were important for the restriction of N-tropic Murine Leukemia Virus. Here, we examined whether SUMO expression and the SIM1 and SIM2 motifs in rhesus monkey TRIM5a (rhTRIM5a) are similarly important for Human Immunodeficiency Type 1 (HIV-) restriction.

Results: We found that mutation of SIM1 and SIM2 of rhTRIM5a abolished the restriction of HIV-1 virus. Further, knockdown of SUMO-1 in rhTRIM5a expressing cells abolished restriction of HIV-1. These results may be due, in part, to the ability of SUMO-1 to stabilize rhTRIM5a protein expression, as SUMO-1 knockdown increased rhTRIM5a turnover and the mutations in SIM1 and SIM2 led to more rapid degradation than the wild type protein. The NF-KB signaling ability of rhTRIM5a was also attenuated by SUMO-1 knockdown. Finally, upon inhibition of CRM1-dependent nuclear export with Leptomycin B (LMB), wild type rhTRIM5a localized to SUMO-1 bodies in the nucleus, while the SIM1 and SIM2 mutants did not localize to SUMO-1.

Conclusions: Our results suggest that the rhTRIM5a B30.2/SPRY domain is not only important for the recognition of the HIV-1 CA, but it is also important for its association with SUMO-1 or SUMO-1 modified proteins. These interactions help to maintain TRIM5a protein levels and its nuclear localization into specific nuclear bodies.
\end{abstract}

Keywords: SUMO-1, SIM, rhTRIM5a, HIV-1

\section{Background}

TRIM5 $\alpha$ is a member of the TRIpartite Motif (TRIM) family of proteins, characterized as having three domains: a RING domain, either one or two B-box domains, and a coiled-coil domain (RBCC) [1]. TRIM5 $\alpha$ is capable of restricting retroviral infection in a species-specific manner. This restriction requires an interaction between the retroviral capsid (CA) lattice and the B30.2/SPRY domain of TRIM5 $\alpha[2-6]$. The B30.2/SPRY domain, located at the Cterminal of TRIM $5 \alpha$, confers the restriction spectrum of TRIM5 $\alpha$ proteins [6]. Human TRIM5 $\alpha$ (huTRIM5 $\alpha$ )

\footnotetext{
*Correspondence: ecampbell@lumc.edu; gloria.arriagada@unab.cl ${ }^{\dagger}$ Equal contributors

'Department of Microbiology and Immunology Stritch School of Medicine, Loyola University Chicago, 2160 S. 1st Ave. 508, Building 102, Room 5651, Maywood, IL 60153, USA

${ }^{2}$ Department of Biochemistry and Molecular Biophysics, Columbia University, 701W168th street, HHSC1310, New York, NY 10032USA

Full list of author information is available at the end of the article
}

potently restricts $\mathrm{N}$-tropic murine leukemia virus (N-MLV) but it does not restrict B-tropic or NB-tropic MLV (B-MLV, NB-MLV respectively) [3,7]. On the other hand, rhesus macaque TRIM5 $\alpha$ (rhTRIM5 $\alpha$ ) restricts N-MLV and human immunodeficiency type 1 (HIV-1) $[6,8]$.

Small ubiquitin-related modifier (SUMO) proteins are conjugated to cellular substrates and regulate diverse cellular processes (for review see $[9,10]$ ). SUMO proteins are transferred to lysine residues within the UBC9 binding site of the target protein. This binding site has a consensus sequence $\Psi$ KXE (where $\Psi$ is a hydrophobic residue, $\mathrm{K}$ is the lysine to which SUMO-1 is conjugated, $\mathrm{X}$ is any amino acid and $\mathrm{E}$ is glutamic acid) [11,12]. Conjugation of SUMO proteins to a substrate mediates distinct protein-protein interactions in vivo. These non-covalent interactions with SUMO modified proteins are mediated by SUMO interacting motifs (SIMs) $[11,13,14]$. The best-characterized SIMs have the consensus sequence $\mathrm{V} / \mathrm{I} / \mathrm{L}-\mathrm{x}-\mathrm{V} / \mathrm{I} / \mathrm{L}-\mathrm{V} / \mathrm{I} / \mathrm{L}$ or $\mathrm{V} / \mathrm{I} /$ $\mathrm{L}-\mathrm{V} / \mathrm{I} / \mathrm{L}-\mathrm{x}-\mathrm{V} / \mathrm{I} / \mathrm{L}$ (where $\mathrm{x}$ is any amino acid) $[14,15]$.

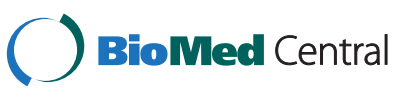

(c) 2013 Lukic et al.; licensee BioMed Central Ltd. This is an Open Access article distributed under the terms of the Creative Commons Attribution License (http://creativecommons.org/licenses/by/2.0), which permits unrestricted use, distribution, and reproduction in any medium, provided the original work is properly cited. 
Figure 1 SUMO interacting motifs are important for HIV-1 restriction by rhesus macaque TRIM5a. CRFK, TE671 and HeLa cells were transduced with a retroviral vector encoding wild type rhTRIM5a, SIM1mut, SIM2mut and SIM3mut or an empty vector (EV) control. A. Western blot assay showing the expression of the FLAGtagged rhTRIM5a, using an anti-FLAG antibody, GAPDH was used as a loading control. B-C. Cells stably expressing the various rhTRIM5a proteins or an EV control were infected with an HIV-1 firefly luciferase reporter virus pseudotyped with VSV-G envelope. 48 hours after infection luciferase activity was measured. Representative experiment of four independent experiments for CRFK (B), HeLa (C) and TE671 (D) cells. E. The CRFK cells were infected with an N-MLV firefly luciferase reporter virus pseudotyped with VSV-G envelope. Representative experiment of four independent experiments. Error bars show standard deviation between triplicates.

We have previously identified that SUMO conjugation system is involved in huTRIM5 $\alpha$ - mediated restriction of N-MLV [16]. Three SIMs were identified in the huTRIM5 $\alpha$ B30.2/SPRY domain. Two of them, SIM1 and SIM2, were responsible for the enhanced restriction of N-MLV in human cells observed upon SUMO-1 overexpression. Specifically, mutation of SIM1 and SIM2 resulted in a dramatic decrease in the ability of huTRIM $5 \alpha$ to restrict $\mathrm{N}-\mathrm{MLV}$ infection. The SIMs are conserved among TRIM5 $\alpha$ orthologs, as the same SIM mutations also affected the rhTRIM5 $\alpha$ restriction of N-MLV [16]. As such, we hypothesized that rhTRIM5 $\alpha$ restriction of HIV-1 was similarly dependent on SUMO-1 expression and the SIM1 and SIM2 motifs of rhTRIM5 $\alpha$.

Here we report that SIMs are important for HIV-1 restriction, and that knockdown of SUMO-1 in rhTRIM5 $\alpha$ expressing cells drastically reduces HIV-1 restriction. We hypothesize that the presence of SUMO-1 stabilizes proteins levels of rhTRIM5 $\alpha$, as knockdown of SUMO-1 decreases steady state levels of rhTRIM $5 \alpha$ and NF- $\mathrm{kB}$ activation. Additionally, mutation of the SIMs abolishes the co-localization of rhTRIM5 $\alpha$ with promyelocytic leukemia protein (PML, also known as TRIM19)/SUMO-1 in the nucleus upon LMB treatment. Our results suggest that the rhTRIM5 $\alpha$ B30.2/SPRY domain is not only important for the recognition of the HIV-1 CA, but that it is also important for its association with SUMO-1 or with SUMO-1 modified proteins. This association helps maintain TRIM5 $\alpha$ protein levels, as well as its ability to mediate NF- $\mathrm{kB}$, and nuclear localization into specific nuclear bodies.

\section{Results and discussion}

Mutations in rhTRIM5a SIM1 and SIM2 motifs abolish HIV-1 restriction

To explore the importance of rhTRIM5 $\alpha$ SIMs in restriction of HIV-1, we generated CRFK, HeLa, and TE671 cell lines stably expressing comparable levels of FLAG-tagged wild type rhTRIM5 $\alpha$ or the rhTRIM5 $\alpha$ variants with 
mutations in SIM1 (376-379), SIM2 (405-408) and SIM3 (430-433) (Figure 1A). These cell lines were infected with VSV-G pseudotyped HIV-1 carrying a firefly luciferase reporter gene to assess retroviral restriction. The wild type and SIM3 rhTRIM5 $\alpha$ efficiently restricted HIV-1 infection when compared to the empty vector (EV) in CRKF (Figure 1B) and HeLa cells (Figure 1C). Conversely, mutation of SIM1 and SIM2 of rhTRIM5 $\alpha$ completely abolished the restriction activity in these cell lines (Figure 1B, C). Similar results were observed in TE671 cells (Figure 1D), although the expression of the SIM1 and SIM2 mutants was noticeably reduced compared to the expression of wild type rhTRIM5 $\alpha$ (Figure 1A). In all cell lines, mutation of rhTRIM5 $\alpha$ lysine 10 to arginine (K10R), a predicted SUMOylation site had minimal effect on restriction, consistent with our previous observations of huTRIM5 $\alpha$ and rhTRIM5 $\alpha$ N-MLV restriction [16]. Similarly, in CRFK cells, which do not express a functional TRIM5 gene [17], the restriction of N-MLV by rhTRIM5 $\alpha$ required a functional SIM1 and SIM2, recapitulating the restriction profile observed for HIV-1 (Figure 1E). Therefore, SIM1 and SIM2 present in rhTRIM5 $\alpha$ are important for its antiviral activity against both N-MLV and HIV-1. Consistent with this observation, another group has recently reported that the SIM1 and SIM2 mutations disrupt the binding of rhTRIM5 $\alpha$ to the HIV-1 capsid [18].

\section{Restriction of HIV-1 by rhTRIM5a is reduced following SUMO-1 knockdown}

To determine if the ability of rhTRIM5 $\alpha$ to restrict HIV-1 infection was dependent on interactions with SUMO-1, we stably knocked down SUMO-1 (SUMO-1 KD) using a SUMO-1 specific shRNA in TE671 cells expressing FLAGrhTRIM5 $\alpha$ or empty vector. A non-silencing shRNA was used as a control. To confirm SUMO-1 KD, we performed quantitative PCR (qPCR) and found that these cells had $\sim 70 \%$ SUMO-1 KD (Figure 2B). Control cells transduced with empty vector did not show appreciable differences in HIV-1 infection following SUMO-1 KD (Figure 2A). Cells transduced to express FLAG-rhTRIM5 $\alpha$ showed considerable restriction of HIV-1. Notably, following SUMO-1 KD the restriction activity of cells expressing FLAG-rhTRIM5 $\alpha$ was reduced to levels similar to cells expressing empty vector (Figure $2 \mathrm{~A}$ ). This demonstrates that rhTRIM5 $\alpha$ restriction of HIV-1 is sensitive to SUMO-1 depletion.

\section{SUMO-1 enhances rhTRIM5a stability in cells}

As noted earlier, TE671 cells expressing rhTRIM5 $\alpha$ SIM mutants showed reduced expression compared to TE671 cell lines expressing wild type or K10R forms of rhTRIM5 $\alpha$ (Figure 1A). This suggests that disrupting interactions with SUMO-1 may increase the turnover of rhTRIM5 $\alpha$. It was previously reported that knockdown of host cellular proteins which interact with rhTRIM5 $\alpha$ increased rhTRIM5 $\alpha$

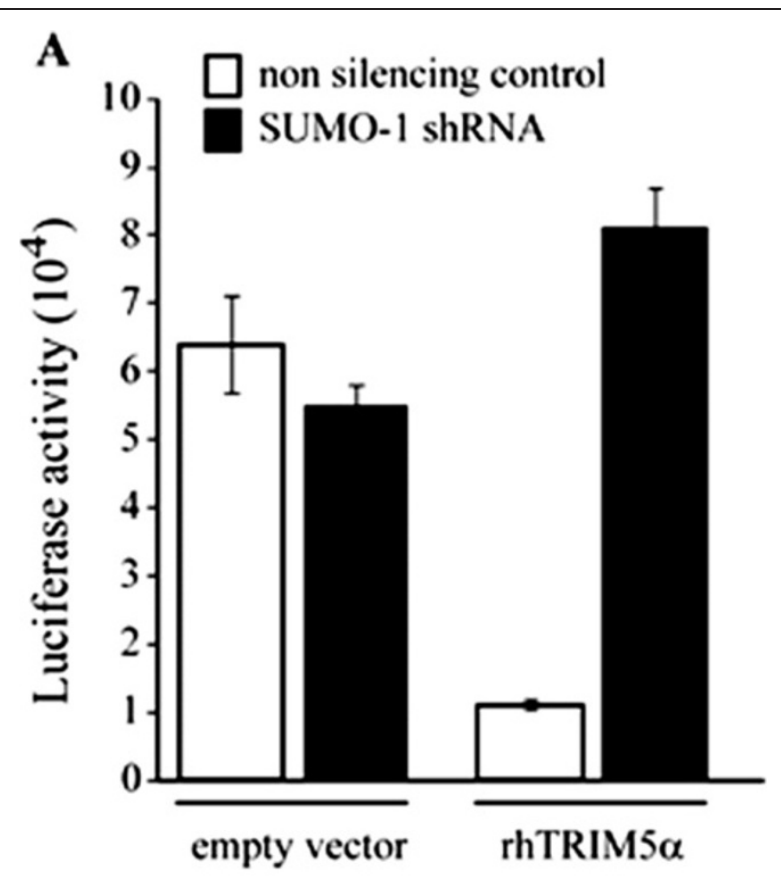

B

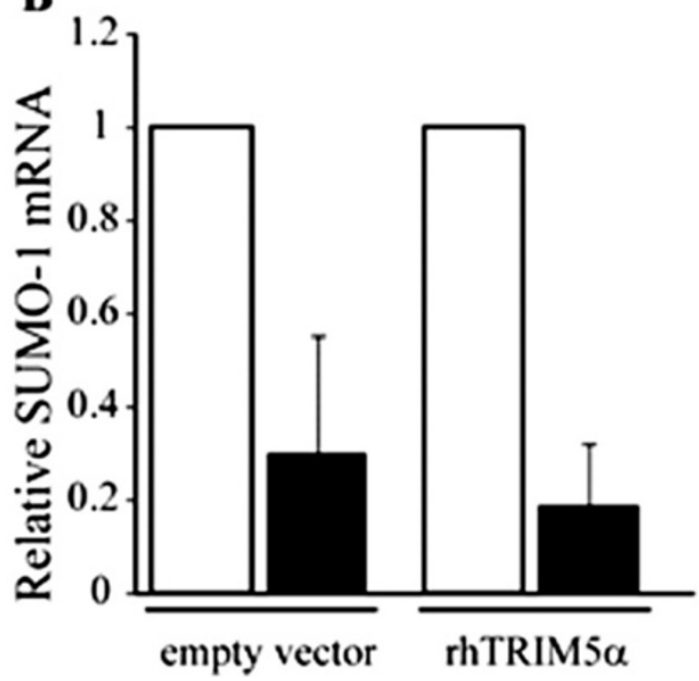

Figure 2 Restriction of HIV-1 by rhTRIM5a is reduced following SUMO-1 knockdown. The TE671 empty vector control cell line or the FLAG-rh TRIM5a expressing cell line were transduced with a retroviral vector encoding a non-silencing shRNA (white bars), or a SUMO-1 specific shRNA (black bars) and selected by antibiotic resistance. $\mathbf{A}$. The different cell lines were infected with an HIV-1 firefly luciferase reporter virus pseudotyped with VSV-G envelope. 48 hours post infection, luciferase activity was measured. Representative experiment of three independent experiments. Error bars show standard deviation between triplicates. B. RNA was extracted, and the mRNA levels of SUMO-1 were determined by quantitative PCR. The values were normalized to GAPDH mRNA and expressed as fold over the respective non-silencing control. Error bars indicate standard deviation between three different quantifications. 
turnover [19]. We therefore examined rhTRIM5 $\alpha$ expression levels by Western blot following SUMO-1 siRNA treatment in a HeLa cell line stably expressing HArhTRIM5 $\alpha$ [8]. In these cells, SUMO-1 KD reduced HArhTRIM5 $\alpha$ expression (Figure 3 ), although this reduction did not correlate with the degree of restriction observed
(Figure 2B). Other studies have noted that small alterations in TRIM5 $\alpha$ expression do not dramatically affect restriction activity at non-saturating amounts of virus [4,20]. These observations make it difficult to separate the contribution of reduced protein expression and the relief of restriction observed in studies of this type. Therefore, it remains

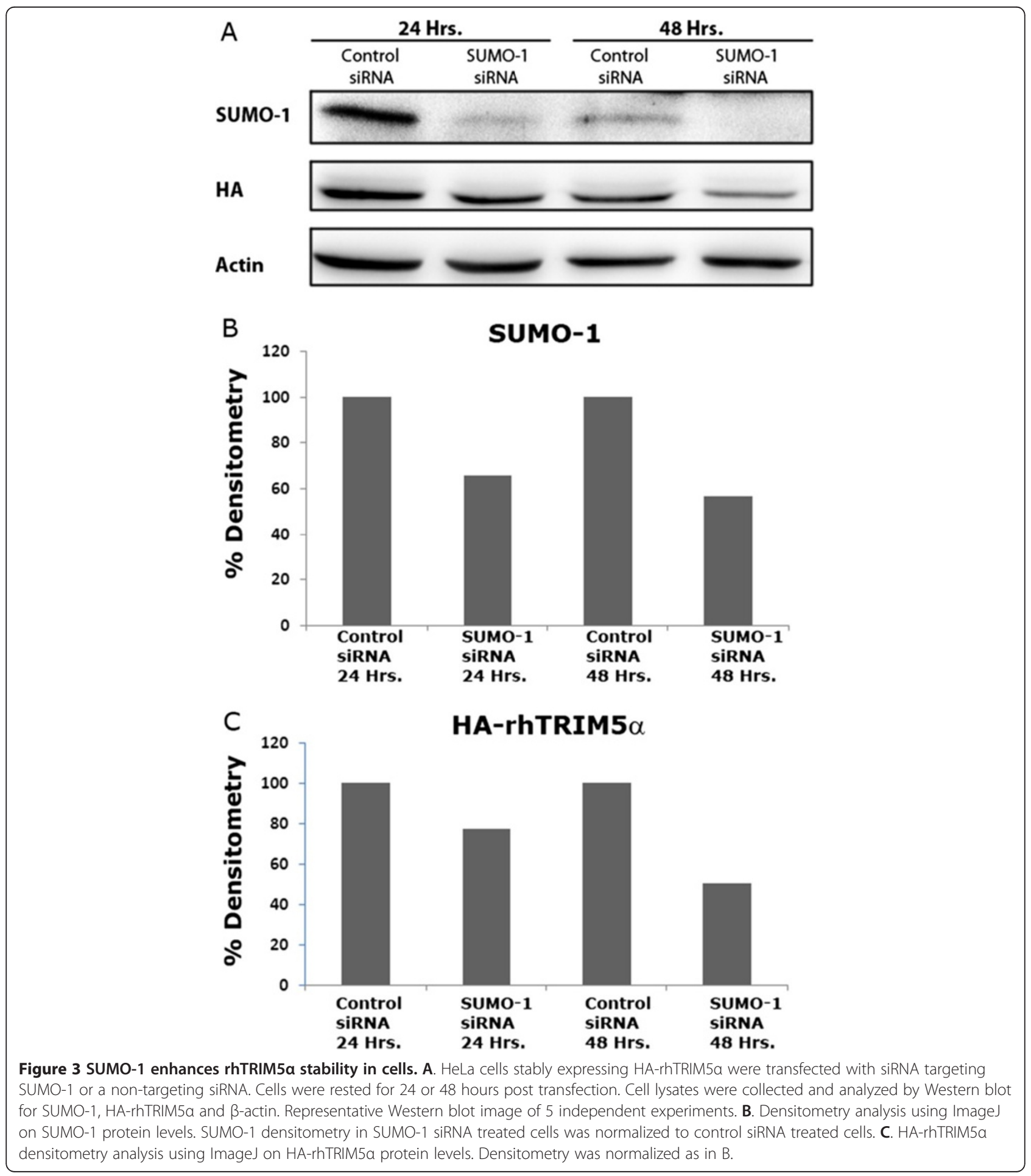


possible that the effects of SUMO-1 KD on restriction are not entirely due to reduced expression of rhTRIM $5 \alpha$.

\section{NF-KB activation by rhTRIM5 $a$ is sensitive to SUMO-1 expression}

Recent studies have shown that TRIM5 proteins can activate intracellular signaling pathways that culminate in AP1 and NF-kB activation [21,22]. In order to understand the role of SUMO-1 and SIMs in rhTRIM5 $\alpha$-mediated signaling, we transiently expressed wild type and $\operatorname{rhTRIM} 5 \alpha$ mutants along with an NF-kB luciferase reporter. Both the wild type and SIM3 mutant form of rhTRIM5 $\alpha$ were able to activate NF-kB. On the other hand, the SIM1 and SIM2 mutants did not induce significant signaling above background in this context (Figure 4A, top panel). However, following transient transfection, the protein expression levels of the SIM1 and SIM2 mutants were reduced compared to wild type and SIM3 mutants, possibly explaining the loss of NF-kB activation (Figure 4A, bottom panel). To assess NF- $\mathrm{kB}$ signaling by rhTRIM $5 \alpha$ SIM mutants at comparable protein levels, we generated 293A cell lines stably expressing wild type rhTRIM5 $\alpha$ and the SIM mutants and measured NF- $\mathrm{kB}$ activation in these cells. Under these conditions, when the SIM mutants were expressed at comparable levels to wild type rhTRIM5 $\alpha$, they elicited similar levels of NF-KB activation (Figure 4B). Consistent with a
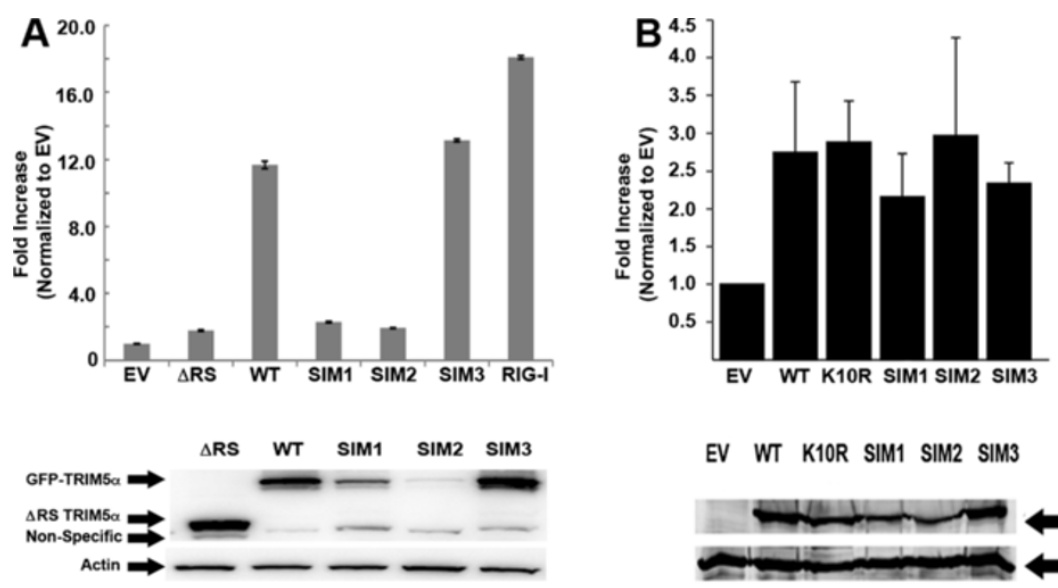

EV WT K10R SIM1 SIM2 SIM3
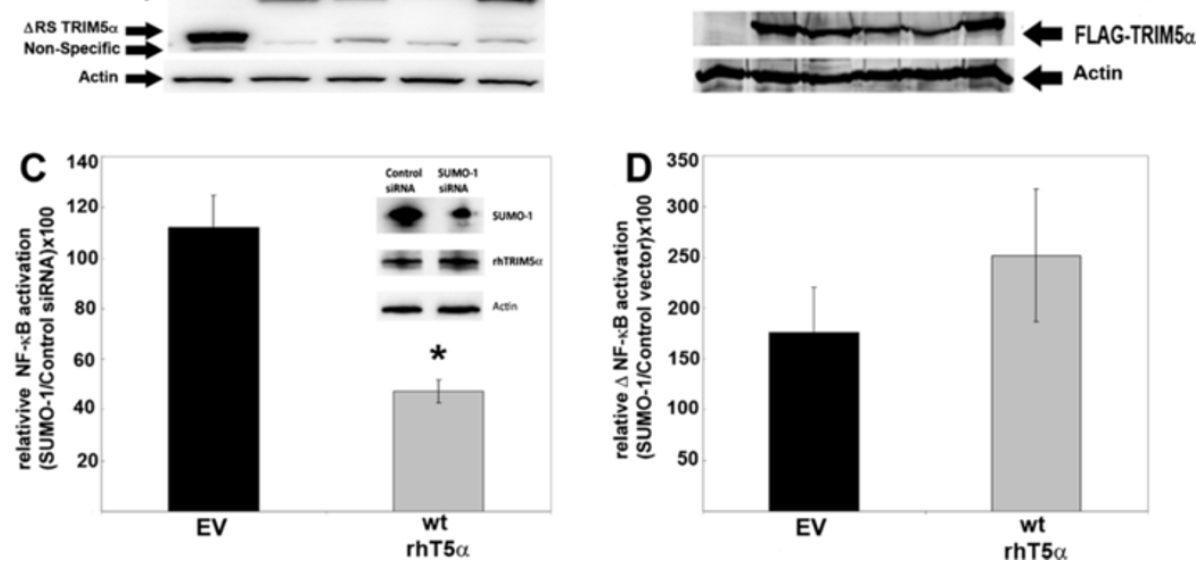

Figure 4 The role of SIMs and SUMO-1 expression on NF-KB activation by rhTRIM5a. A. 293T cells plated in triplicate were transfected with empty vector (EV), $\triangle \mathrm{RING/SPRY}$ rhTRIM5a $(\triangle \mathrm{RS}$ ), wild typerhTRIM5a, SIM mutants or RIG-I along with NF-kB-responsive firefly luciferase construct. A renila luciferase construct was used as an internal transfection efficiency control. 48-hours post transfection, cells were lysed and luciferase activity was measured. NF-KB luciferase readings were normalized to renilla luciferase, and plotted as an average fold increase over empty vector Upper panel, representative luciferase activity in the presence of various constructs.Error bars represent the SEM between the triplicates. Lower panel,representative Western blot. Representative of 4 independent experiments. B. 293A stably expressing wild typerhTRIM5a, K10R mut, SIM mutants with a FLAG-tag or an EV. Cells weretransiently transfected with an NF-KB firefly luciferase and renila luciferase plasmids as in A. Upper panel, NF-KB luciferase activity in the presence of rhTRIM5a mutants. Error bars represent the SD between 3 independent experiments. Lower panel, representative Western blot at the time of data acquisition. C. 293T cells were transfected with Control siRNA or SUMO-1 siRNA for 48 hours. Cells were then seeded in a 96-well plate in triplicate and transfected with empty vector or wild type rhTRIM5a. NF-kB activity was measured as in A. Data were plotted by dividing SUMO-1 siRNA activation by control siRNA activation x 100. Inset, representative Western blot. P $<0.004$ by Student's t-test. Error bars represent the SEM between the triplicates. Data is representative of 3independent experiments. D. 293T cells were transfected with empty vector, and wild type rhTRIM5a constructs in presence and absence of SUMO-1. NF-kB activity was measured as in A. Data were plotted as in C. Error bars represent the SEM between triplicates. Representative of 3 independent experiments. 
previous report that showed normal oligomerization of these mutants [18], and the data here that demonstrate the ability of these mutants to activate NF- $\kappa B$, we conclude that the defect in restriction by rhTRIM $5 \alpha$ SIM mutants is not due to gross misfolding of the protein.

We next asked how SUMO-1 depletion affected the ability of wild type rhTRIM $5 \alpha$ to induce NF-кB activation. We co-transfected $293 \mathrm{~T}$ cells with wild type HArhTRIM5 $\alpha$, SUMO-1 or control siRNA and an NF-кB driven luciferase reporter. We measured NF- $\mathrm{kB}$ activation by rhTRIM5 $\alpha$ in SUMO-1 siRNA treated cells compared to rhTRIM $5 \alpha$ cells treated with control siRNA. As shown in Figure 4C knocking down SUMO-1 had little effect on NF- $\mathrm{KB}$ activation when transfected with EV ( 10\%, black bar). However, depletion of SUMO-1 significantly reduced ( $\sim 60 \%, \mathrm{p}<0.004$, Student's T-test) NF- $\mathrm{kB}$ activation by wild type rhTRIM5 $\alpha$ (Figure $4 \mathrm{C}$, grey bar). The reduction of NF- $\kappa \mathrm{B}$ activation in the presence of SUMO-1 siRNA was not due to reduced rhTRIM5 $\alpha$ protein levels (Figure $4 \mathrm{C}$ inset) as SUMO-1 knockdown does in cells stably expressing rhTRIM5 $\alpha$ (Figure 3). Conversely, overexpression of SUMO-1 increased NF- $\kappa$ B activation following transfection with empty vector or vector expressing wild type rhTRIM5 $\alpha$ (Figure 4D). This increase was more pronounced when rhTRIM5 $\alpha$ was present, although this result was not statistically significant $(\mathrm{p}=0.187$, Student T-test). These experiments demonstrate that the ability to associate with SUMO-1 or SUMOylated proteins is relevant to rhTRIM $5 \alpha$-mediated NF- $\mathrm{kB}$ signaling.
The SIM1 and SIM2 mutations disrupt rhTRIM5a trafficking to nuclear bodies containing PML and SUMO-1 We next analyzed the association of rhTRIM5 $\alpha$ and SUMO-1 by immunofluorescence to verify that SIM1 and SIM2 do not interact with SUMO-1 or SUMO-1 modified proteins. In HeLa cells stably expressing YFP-rhTRIM5 $\alpha$, we examined the co-localization of rhTRIM5 $\alpha$ and endogenous SUMO-1. We used two antibodies to SUMO-1 to examine both the cytoplasmic and nuclear fractions of SUMO-1. The first antibody (GMP1, clone 21C7) recognized nuclear SUMO-1 as well as numerous cytoplasmic puncta. However, the cytoplasmic SUMO-1 did not colocalize with YFP-rhTRIM5 $\alpha$ (Figure 5). When these cells were stained with an antibody to PML, we did not observe significant localization of SUMO-1 to PML (data not shown). As SUMO-1 positive structures in the nucleus are well characterized and known to contain PML [23,24], we used a second antibody (clone Y299) that recognized nuclear structures that were PML positive (Data not shown). This antibody detected primarily diffuse and punctate nuclear SUMO-1. We used this antibody in subsequent experiments to examine SIM1 and SIM2 localization with SUMO-1. A recent study demonstrated that while steady state rhTRIM5 $\alpha$ is excluded from the nucleus, it can transiently enter and exits the nucleus, where it associates with PML bodies. This nuclear localization of rhTRIM5 $\alpha$ to PML bodies is observed when the nuclear export of rhTRIM $5 \alpha$ is inhibited with Leptomycin B (LMB), which is an inhibitor of CRM1 mediated nuclear export [25].
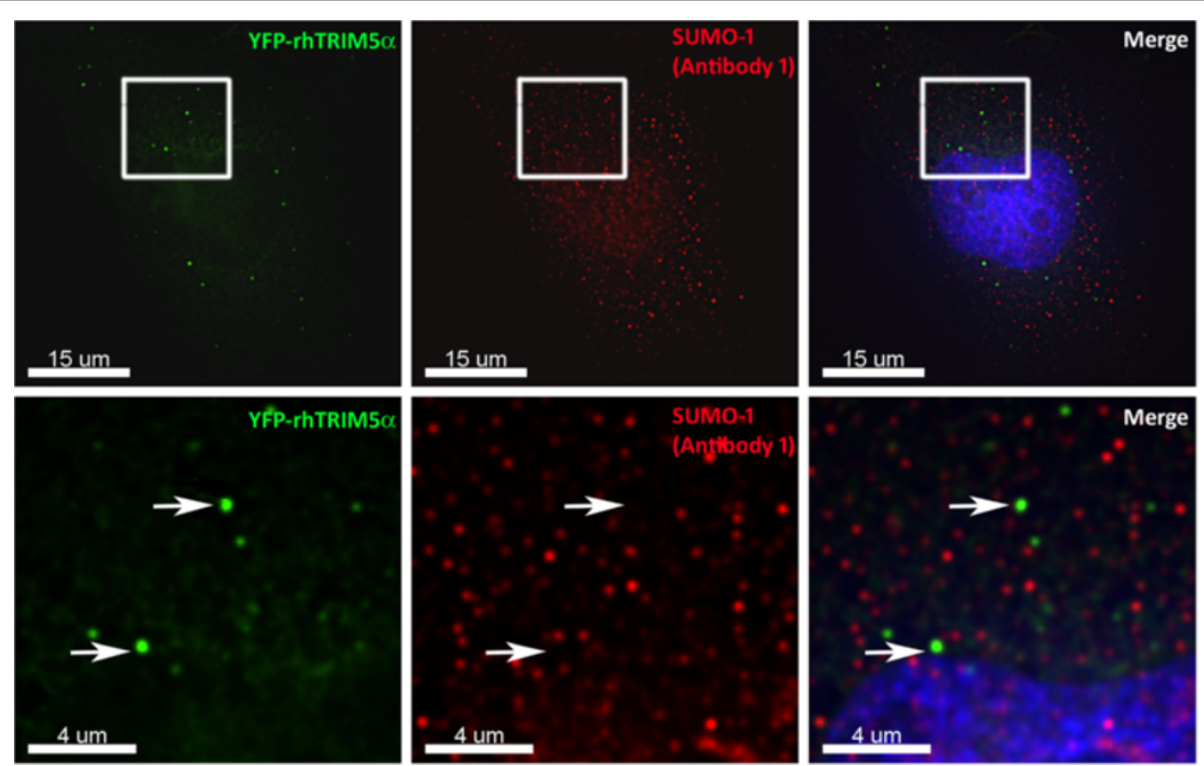

Figure 5 SUMO-1 antibody recognizing cytoplasmic SUMO-1 does not localize to rhTRIM5a cytoplasmic bodies. HeLa cells stably expressing YFP-rhTRIM5a were immunostained with a mouse anti-SUMO-1 (GMP1) clone 21C7. The white box in the top panel represents the area that was zoomed in to create the bottom panel. White arrows point to representative rhTRIM5a cytoplasmic bodies that do not contain SUMO-1. 


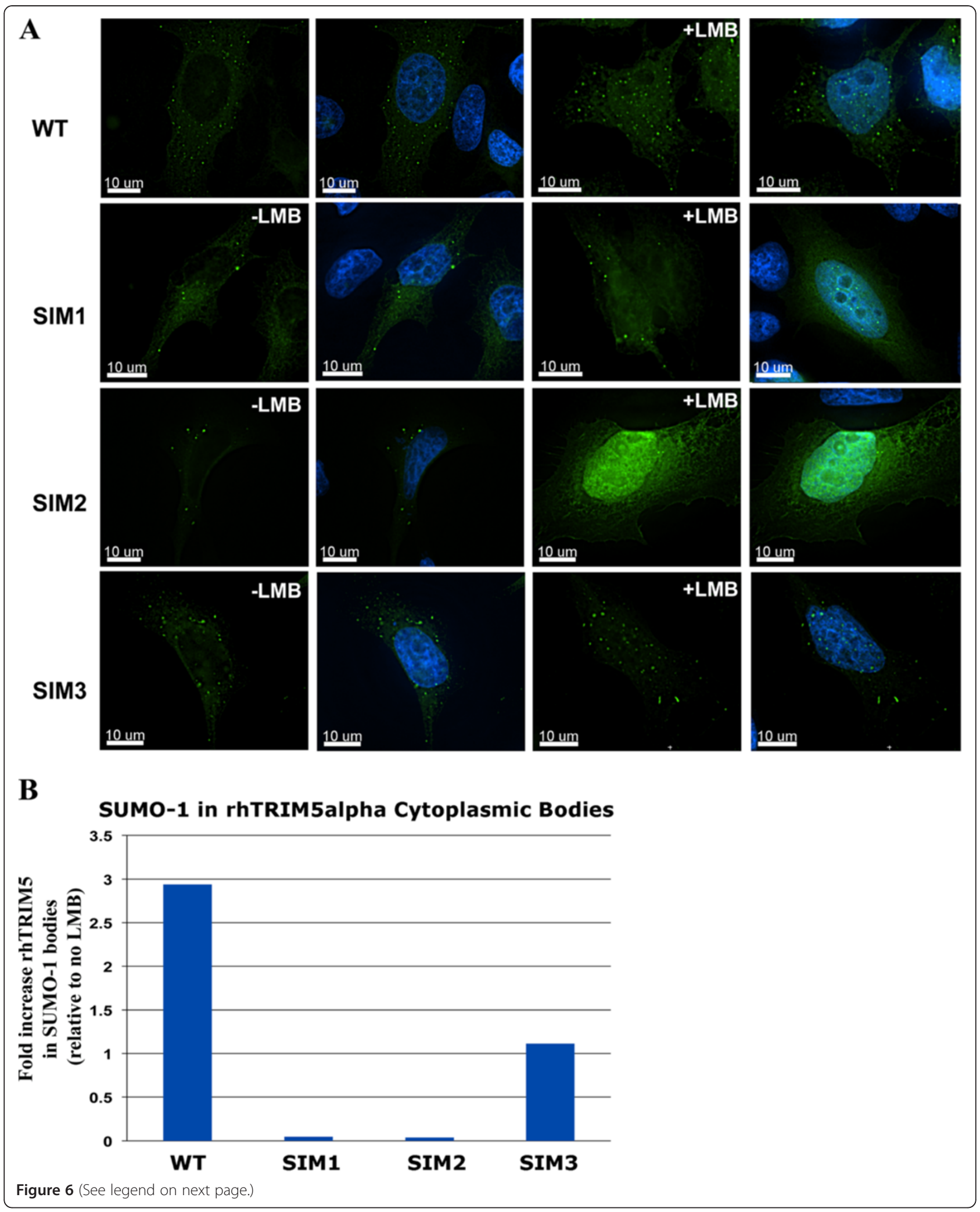




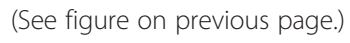

Inhibiting the nuclear export of rhTRIM5 $\alpha$ using LMB revealed that wt and all three SIM mutants localized to the nucleus (Figure 6A), contrary to a recent report by another group which found that the SIM1 and SIM2 mutants did not localize to the nucleus under these conditions [18]. However, when we quantified the localization of these mutants to nuclear SUMO-1 bodies, SIM1 and SIM2 mutants of rhTRIM $5 \alpha$ failed to localize to SUMO-1 positive bodies when nuclear export is inhibited, while the SIM3 mutant associated with these bodies to an intermediate degree (Figure 6B). These SUMO-1 positive rhTRIM5 $\alpha$ nuclear bodies were also positive for PML (data not shown).

\section{Potential mechanisms of SUMO-1 regulation of rhTRIM5a} Collectively, the data presented here demonstrate that SIMs in rhTRIM5 $\alpha$ and SUMO-1 are important for rhTRIM5 $\alpha$ mediated retroviral restriction. rhTRIM5 $\alpha$ is sensitive to changes in SUMO-1 expression as SUMO-1 knockdown relieves rhTRIM5 $\alpha$ restriction (Figure 2) and increases rhTRIM5 $\alpha$ protein turnover in stable cells (Figure 3). Additionally, SUMO-1 modulates rhTRIM5 $\alpha$-dependent activation of NF-kB (Figure 4). Upon SUMO-1 knockdown, NF- $\kappa B$ activation is decreased in a rhTRIM5 $\alpha$-dependent manner (Figure 4C). We also observed decreased localization of SIM1 and SIM2 to SUMO-1 puncta following LMB treatment (Figure 6).

The mechanism by which SUMO-1 stabilizes rhTRIM5 $\alpha$ expression or affects retroviral restriction is unclear. It is possible that rhTRIM5 $\alpha$ interacts with a protein that stabilizes its expression in a SUMO-dependent manner. It is also possible that the expression of another cellular protein that is stabilized by SUMOylation, stabilizes rhTRIM5 $\alpha$ expression. Consistent with this, it is known that SUMOylation can protect some proteins from ubiquitin dependent degradation [26]. Our observation that the SIM1 and SIM2 mutations, which abrogate restriction, are also turned over more rapidly than wild type rhTRIM5 $\alpha$ are consistent with all of these possibilities. Interestingly, a recent study by Brandariz-Nunez et al. suggests that these residues are not present on the surface of the SPRY domain [18], as modeled using an NMR structure recently described for the PRY/SPRY domain [27]. It may be that conformational changes induced by the binding of other cellular factors conditionally expose the SIM1 and SIM2 motifs. These authors also observe that SIM1 and SIM2 mutants oligomerize normally [18], suggesting these proteins are not misfolded. While we cannot exclude the possibility that SIM1 and SIM2 mutations prevent proper folding of the B30.2/SPRY domain, our own results demonstrate that alterations in SUMO-1 expression can influence the stability of wild type rhTRIM $5 \alpha$.

The data presented here may begin to explain the mechanistic of rhTRIM5 $\alpha$ nuclear trafficking. It is possible that the recruitment of TRIM5 $\alpha$ to SUMO-1/PML bodies has direct relevance to the restriction process, as both the SIM1 and SIM2 mutants that do not localize to SUMO-1/PML bodies are completely unable to restrict retroviral infection. Considering that PML protein undergoes SUMOylation on three lysine residues [28-30] and rhTRIM5 $\alpha$ has a rapid turnover [31], we can speculate that the steady state levels of rhTRIM5 $\alpha$ protein are maintained by its interaction via SIMs with SUMOylated-PML in the nucleus. When SIMs or SUMO-1 are not present rhTRIM5 $\alpha$ is less retained in the nucleus and therefore is degraded faster in the cytosol, reducing the amount of protein available to restrict incoming viruses.

Interestingly, owl monkey TRIM-Cyp, which contains a C-terminal cyclophilin A domain instead of a B302/SPRY domain (the B30.2/SPRY domain contains all three putative SIMs) [32], does not traffic to the nucleus [25]. Therefore, given the strong homology between the RBCC domains of owl monkey TRIM-Cyp and rhTRIM $5 \alpha$, it is possible that the SPRY domain contains the determinants that govern nuclear trafficking. Consistent with this hypothesis, our data demonstrate that wild type rhTRIM5 $\alpha$, but not the SIM1 and SIM2 mutants, localizes to nuclear SUMO-1 bodies (Figure 5) support this idea. Although the results we have obtained using the SIM1 and SIM2 mutants were consistent with the effect of SUMO-1 knockdown on wild type rhTRIM $5 \alpha$ activity, the results obtained with these mutants should be cautiously interpreted [18].

Future studies are needed to determine if this association is merely correlative or the association of rhTRIM $5 \alpha$ with nuclear SUMO-1/PML bodies potentiates specific steps in the restriction process.

\section{Conclusions}

Our results demonstrate that rhTRIM5 $\alpha$ restriction and expression is governed by SUMO-1 expression. SUMO-1 depletion, or mutations that are expected to disrupt 
SUMO-1 binding, abrogate restriction and increase the rate of rhTRIM $5 \alpha$ turnover. The ability of rhTRIM $5 \alpha$ to activate innate immune signaling pathways is also sensitive to SUMO-1 expression. Additionally, the ability to traffic to nuclear SUMO-1 bodies is abrogated by mutation of the SIM1 or SIM2 motifs present in rhTRIM5 $\alpha$. As rhTRIM5 $\alpha$ does not appear to itself be SUMOylated, future studies are needed to identify the cellular factor or factors that are likely regulating rhTRIM5 $\alpha$ in a SUMO-1 dependent fashion.

\section{Methods}

\section{Cell lines and plasmids}

Human embryonic fibroblast 293T, 293A, human medulloblastoma cell line TE671, HeLa and Crandall feline kidney (CRFK) fibroblast were maintained in Dulbecco's modified Eagle medium supplemented with $10 \%$ fetal bovine serum, $100 \mathrm{UI} / \mathrm{ml}$ penicillin and $100 \mathrm{mg} / \mathrm{ml}$ streptomycin. All cells were cultured at $37^{\circ} \mathrm{C}$ in $5 \% \mathrm{CO}_{2}$.

The rhTRIM $5 \alpha$ constructs encoding the FLAG tagged wild type and mutant versions were identified and cloned as previously described [16]. YFP tagged wild type and mutant versions were cloned into a retroviral vector previously described [33]. RIG-I construct was kindly donated to us by Dr. Susan Baker (Loyola University Chicago).

\section{Generation of stable cell lines}

Retroviruses for transduction were produced by transfection of $293 \mathrm{~T}$ cells with $1 \mu \mathrm{g}$ pMD.G, $1 \mu \mathrm{g}$ pCMVI and 1.5 $\mu \mathrm{g}$ of either pQCXIN, pQCXIN-FLAG-rhTRIM5 $\alpha$ wildtype or mutant versions, using FUGENE (Roche). Viruses were harvested $48 \mathrm{~h}$ after transfection, filtered $(0.45 \mu \mathrm{m})$ and used to infect $5 \times 10^{5}$ cells in $100 \mathrm{~mm}$ dishes in the presence of $8 \mu \mathrm{g} / \mathrm{ml}$ polybrene. HeLa, TE671, 293A cells infected with vectors delivering the $\mathrm{Neo}^{\mathrm{r}}$ gene were selected in $1.5 \mu \mathrm{g} / \mathrm{ml}$ G418. The CRFK cells overexpressing rhTRIM5 $\alpha$ were previously described [16]. Lentiviruses for transduction were produced by transfection of 293T cells with $1 \mu \mathrm{g}$ pMD.G, $1 \mu \mathrm{g}$ p8.91 and $1.5 \mu \mathrm{g}$ of pGIPz (Open Biosystems) or pGIPzSUMO-1 DNAs containing shRNA (Open Biosystems). Viruses were harvested $48 \mathrm{~h}$ after infection, filtered $(0.45 \mu \mathrm{m})$ and used to infect $5 \times 10^{4}$ TE671-rhTRIM5 $\alpha$ cells in $35 \mathrm{~mm}$ dishes in the presence of $8 \mu \mathrm{g} / \mathrm{ml}$ polybrene. Cells were selected in 1.5 $\mu \mathrm{g} / \mathrm{ml} \mathrm{G} 418$ and $1.5 \mu \mathrm{g} / \mathrm{ml}$ puromycin.

\section{Western blotting}

Cells were lysed in 20 mMTris- $\mathrm{HCl}$ (pH 8.0), 137 mMKCl, 10\% glycerol, 1\% NP-40 and complete protease inhibitor (Roche) or Reporter lysis buffer (Promega). Samples were then boiled in $5 \mathrm{x}$ sodium dodecyl sulphate (SDS) loading buffer, and the proteins were resolved by SDS-polyacrylamide gel electrophoresis (PAGE). After transfer to nitrocellulose membranes, the blots were probed with mouse anti- $\beta$ actin (Sigma), anti-HA (Covance), anti SUMO-1 (Abcam clone Y299), anti-FLAG (Sigma) or anti-GAPDH (Calbiochem).

\section{Single-cycle infectivity assay}

HIV-1 luciferase reporter virus was produced by transfection of $293 \mathrm{~T}$ cells with $2 \mu \mathrm{g}$ pNL4.3-env luciferase, and $1 \mu \mathrm{g}$ pMD.G (per $100 \mathrm{~mm}$ plate) using FUGENE (Roche). Reporter virus stocks were harvested $48 \mathrm{~h}$ after transfection, then filtered $(0.45 \mu \mathrm{m})$ and stored at $-80^{\circ} \mathrm{C}$. 293A $\left(3 \times 10^{4}\right.$ per well), HeLa $\left(2.5 \times 10^{4}\right.$ per well), TE671 $\left(2.5 \times 10^{4}\right.$ per well $)$ and CRFK $\left(3 \times 10^{4}\right.$ per well) cells were seeded in 24-well plates and infected with MLV luc reporter viruses. Fortyeight hours post-infection cells were collected and assayed for firefly luciferase activity (Promega) in a luminometer.

\section{Analysis of SUMO-1 knock down}

In the case of the TE671 cells where SUMO-1 was stably knocked down, the cells were harvested and total RNA was extracted using TRIZOL reagent (Invitrogen). $2 \mu \mathrm{g}$ of total RNA per cell line were used to produce cDNA using random hexamers and SuperScript III kit (Invitrogen). $2 \mu \mathrm{l}$ of each cDNA were used for quantitative PCR analysis of SUMO-1 and GAPDH transcript levels. Fold change was calculated using the relative standard curve method.

For the transient knockdown of SUMO-1, HeLa cells stably expressing HA-rhTRIM5 $\alpha$ were transfected with siRNA specifically targeting SUMO-1 or a non-targeting siRNA (Santa Cruz Biotechnology). Cells were transfected with Lipofectamine2000 (Invitrogen) using a 2-day transfection protocol followed by 24-hours or 48-hours of rest to gain maximum knockdown efficiency. Cells were collected following the resting period and analyzed by western blot using a monoclonal anti-SUMO-1 antibody (Abcam clone Y299). Densitometry analysis was performed on the western blots using ImageJ software.

\section{Immunofluorescence}

HeLa cells transfected with wild type YFP-rhTRIM5 $\alpha$ and SIM mutants (Lipofectamine2000 protocol) were allowed to adhere to fibronectin-treated glass coverslips. Cells were treated with LMB for 4 hours and fixed with $3.7 \%$ formaldehyde (Polysciences) in 0.1 M PIPES, pH 6.8 [piperazine$N, \quad N$-bis(2-ethanesulfonic acid)] (Sigma). Monoclonal rabbit anti-SUMO-1 (Abcam clone Y299) antibody was used to stain SUMO-1. Primary antibody was secondarily labeled with Cy5 fluorophore-conjugated donkey antimouse antibody (Jackson ImmunoResearch). Nucleus was stained using a DAPI stain (Jackson ImmunoResearch). Images were collected with a DeltaVision microscope (Applied Precision) equipped with a digital camera (CoolSNAP HQ; Photometrics), using a 1.4-numerical aperture 100× objective lens, and they were deconvolved with SoftWoRx deconvolution software (Applied Precision). 


\section{Image analysis}

20 Z-stack images were acquired using identical acquisition parameters. Surfaces for cytoplasmic bodies in all samples analyzed were defined by using a fluorescence threshold for YFP-rhTRIM5 $\alpha$, and all YFP-rhTRIM5 $\alpha$ bodies over a volume of $0.011 \mu \mathrm{m}^{3}$ were used in the analysis. Deconvolved images were analyzed for SUMO-1 maximum fluorescence intensity (MFI) in cytoplasmic bodies using the Surface Finder function of the Imaris software package (Bitplane) and the data was plotted in Prism (Graphpad Software Inc) for statistical analysis.

\section{Dual-luciferase reporter assay \\ SIMs}

293T cells seeded in a 96-well plate were transfected with empty vector, $\triangle$ RING/SPRY rhTRIM5 $\alpha$ ( $\triangle \mathrm{RS}$, negative control), wild type rhTRIM5 $\alpha$, SIM1 mut, SIM2 mut, SIM3 mut or RIG-1 (positive control) in triplicate. Transfection was carried out using polyethylenimine (PEI) protocol in which the constructs were added at a $9(\mathrm{EV} /$ rhTRIM5 $\alpha /$ RIG-I): 3 (NF-kB-responsive firefly luciferase construct): 1 (Renilla luciferase construct for transfection efficiencies) ratio. Cells were lysed 48-hours post transfection with Passive lysis buffer (Promega) and the luciferase activity was measured using a Dual-Glo luciferase assay system (Promega) in a Veritas Microplate luminometer. Firefly luciferase data were normalized to Renilla luciferase readings in each well. Data were plotted by determining the fold increase over empty vector.

\section{SUMO-1 overexpression}

293T cells seeded in a 96-well plate were transfected with empty vector, and rhTRIM5 $\alpha$ constructs in presence or absence of SUMO-1 in triplicate. Transfection was carried out using PEI protocol in which the constructs were added at a 5 (rhTRIM5 $\alpha): 4$ (SUMO-1 or EV): 3 (NF-кB-responsive firefly luciferase construct): 1 (Renilla luciferase construct for normalization of transfection efficiencies) ratio. Cells were lysed 48-hours post transfection with Passive lysis buffer (Promega) and the luciferase activity was measured using a Dual-Glo luciferase assay system (Promega) in a Veritas Microplate luminometer. Firefly luciferase data were normalized to Renilla luciferase readings in each well. Data were plotted by dividing SUMO-1 siRNA NF$\kappa B$ activation by Control siRNA NF- $\kappa B$ activation $x 100$.

\section{SUMO-1 knockdown}

293T cells seeded in a 12-well plate were transfected with Control siRNA or SUMO-1 siRNA (Santa Cruz Biotechnology, Inc) following a Lipofectamine2000 (Invitrogen) protocol for two days. On the third day the cells were seeded in a 96-well plate in triplicate and transfected with empty vector or wild type rhTRIM5 $\alpha$ using PEI in which constructs were added at a 9 (EV/rhTRIM5 $\alpha)$ : 3 (NF-kB- responsive firefly luciferase construct): 1 (Renilla luciferase construct for transfection efficiencies) ratio. Cells were lysed 48-hours post transfection with Passive lysis buffer (Promega) and the luciferase activity was measured using a Dual-Glo luciferase assay system (Promega) in a Veritas Microplate luminometer. Firefly luciferase data were normalized to Renilla luciferase readings in each well. Data were plotted by dividing SUMO-1 siRNA NF-kB activation by Control siRNA NF- $\mathrm{kB}$ activation $\mathrm{x} 100$.

\section{Abbreviation \\ SUMO: Small ubiquitin-related protein; SIM: SUMO-interacting motif; rhTRIM5a: Rhesus monkey TRIM5a; HIV-1: Human immunodeficiency virus; KD: Knocked down.}

\section{Competing interests}

The authors do not have competing interests.

\section{Authors' contributions}

GA designed the research and performed experiments. ZL designed and performed experiments. EMC designed experiments and analyzed the data. SPG discussed and analyzed the data. GA and EMC wrote the manuscript, and all authors read the manuscript and added their corrections. All authors read and approved the final manuscript.

\section{Acknowledgements}

GA was a Pew Latin American Fellow. SPG is a Howard Hughes Medical Institute Investigator. EMC is supported by National Institutes of Health grant R01 Al093258.

\section{Author details}

'Department of Microbiology and Immunology Stritch School of Medicine, Loyola University Chicago, 2160 S. 1st Ave. 508, Building 102, Room 5651, Maywood, IL 60153, USA. ²Department of Biochemistry and Molecular Biophysics, Columbia University, 701W168th street, HHSC1310, New York, NY 10032USA. ${ }^{3}$ Howard Hughes Medical institute. ${ }^{4}$ Departamento de Ciencias Biologicas, Facultad de Ciencias Biologicas, Universidad Andres Bello, Los Fresnos 52, Viña del Mar, Chile.

Received: 16 September 2012 Accepted: 16 January 2013

Published: 1 February 2013

\section{References}

1. Nisole S, Stoye JP, Saib A: TRIM family proteins: retroviral restriction and antiviral defence. Nat Rev Microbiol 2005, 3:799-808.

2. Nakayama EE, Miyoshi H, Nagai $Y$, Shioda T: A specific region of 37 amino acid residues in the SPRY (B30.2) domain of African green monkey TRIM5alpha determines species-specific restriction of simian immunodeficiency virus SIVmac infection. J Virol 2005, 79:8870-8877.

3. Perron MJ, Stremlau M, Song B, UIm W, Mulligan RC, Sodroski J: TRIM5alpha mediates the postentry block to N-tropic murine leukemia viruses in human cells. Proc Natl Acad Sci U S A 2004, 101:11827-11832.

4. Sebastian S, Luban J: TRIM5alpha selectively binds a restriction-sensitive retroviral capsid. Retrovirology 2005, 2:40.

5. Stremlau M, Perron M, Lee M, Li Y, Song B, Javanbakht H, Diaz-Griffero F, Anderson DJ, Sundquist WI, Sodroski J: Specific recognition and accelerated uncoating of retroviral capsids by the TRIM5alpha restriction factor. Proc Natl Acad Sci U S A 2006, 103:5514-5519.

6. Stremlau M, Perron M, Welikala S, Sodroski J: Species-specific variation in the B30.2(SPRY) domain of TRIM5alpha determines the potency of human immunodeficiency virus restriction. J Virol 2005, 79:3139-3145.

7. Hatziioannou T, Perez-Caballero D, Yang A, Cowan S, Bieniasz PD: Retrovirus resistance factors Ref1 and Lv1 are species-specific variants of TRIM5alpha. Proc Natl Acad Sci U S A 2004, 101:10774-10779.

8. Stremlau M, Owens CM, Perron MJ, Kiessling M, Autissier P, Sodroski J: The cytoplasmic body component TRIM5alpha restricts HIV-1 infection in Old World monkeys. Nature 2004, 427:848-853.

9. Hay RT: SUMO: a history of modification. Mol Cell 2005, 18:1-12. 
10. Geiss-Friedlander R, Melchior F: Concepts in sumoylation: a decade on. Nat Rev Mol Cell Biol 2007, 8:947-956.

11. Minty A, Dumont X, Kaghad M, Caput D: Covalent modification of p73alpha by SUMO-1. Two-hybrid screening with p73 identifies novel SUMO-1-interacting proteins and a SUMO-1 interaction motif. J Biol Chem 2000, 275:36316-36323.

12. Sampson DA, Wang M, Matunis MJ: The small ubiquitin-like modifier-1 (SUMO-1) consensus sequence mediates Ubc9 binding and is essential for SUMO-1 modification. J Biol Chem 2001, 276:21664-21669.

13. Hannich JT, Lewis A, Kroetz MB, Li SJ, Heide H, Emili A, Hochstrasser M: Defining the SUMO-modified proteome by multiple approaches in Saccharomyces cerevisiae. J Biol Chem 2005, 280:4102-4110.

14. Song J, Zhang Z, Hu W, Chen Y: Small ubiquitin-like modifier (SUMO) recognition of a SUMO binding motif: a reversal of the bound orientation. J Biol Chem 2005, 280:40122-40129.

15. Hecker CM, Rabiller M, Haglund K, Bayer P, Dikic I: Specification of SUMO1and SUMO2-interacting motifs. J Biol Chem 2006, 281:16117-16127.

16. Arriagada G, Muntean LN, Goff SP: SUMO-interacting motifs of human TRIM5alpha are important for antiviral activity. PLOS Pathog 2011, 7:e1002019

17. McEwan WA, Schaller T, Ylinen LM, Hosie MJ, Towers GJ, Willett BJ: Truncation of TRIM5 in the Feliformia explains the absence of retroviral restriction in cells of the domestic cat. J Virol 2009, 83:8270-8275.

18. Brandariz-Nunez A, Roa A, Valle-Casuso JC, Biris N, Ivanov D, Diaz-Griffero F: Contribution of SUMO-interacting motifs and SUMOylation to the antiretroviral properties of TRIM5alpha. Virology 2013, 435(2):463-471. doi:10.1016/j.virol.2012.09.042. Epub 2012 Oct 16

19. O'Connor C, Pertel T, Gray S, Robia SL, Bakowska JC, Luban J, Campbell EM: p62/sequestosome- 1 associates with and sustains the expression of retroviral restriction factor TRIM5alpha. J Virol 2010, 84:5997-6006.

20. Sebastian S, Grutter C, Strambio de Castillia C, Pertel T, Olivari S, Grutter MG, Luban J: An invariant surface patch on the TRIM5alpha PRYSPRY domain is required for retroviral restriction but dispensable for capsid binding. J Virol 2009, 83:3365-3373.

21. Pertel T, Hausmann S, Morger D, Zuger S, Guerra J, Lascano J, Reinhard C, Santoni FA, Uchil PD, Chatel L, et al: TRIM5 is an innate immune sensor for the retrovirus capsid lattice. Nature 2011, 472:361-365.

22. Tareen SU, Emerman M: Human Trim5alpha has additional activities that are uncoupled from retroviral capsid recognition. Virology 2011, 409:113-120.

23. Everett RD, Chelbi-Alix MK: PML and PML nuclear bodies: implications in antiviral defence. Biochimie 2007, 89:819-830.

24. Lallemand-Breitenbach V, de The H: PML nuclear bodies. Cold Spring Harb Perspect Biol 2010, 2:a000661.

25. Diaz-Griffero F, Gallo DE, Hope TJ, Sodroski J: Trafficking of some old world primate TRIM5alpha proteins through the nucleus. Retrovirology 2011, 8:38.

26. Praefcke GJ, Hofmann K, Dohmen RJ: SUMO playing tag with ubiquitin. Trends Biochem Sci 2012, 37:23-31.

27. Biris N, Yang Y, Taylor AB, Tomashevski A, Guo M, Hart PJ, Diaz-Griffero F, Ivanov DN: Structure of the rhesus monkey TRIM5alpha PRYSPRY domain, the HIV capsid recognition module. Proc Natl Acad Sci U S A 2012, 109:13278-13283.

28. Matunis MJ, Zhang XD, Ellis NA: SUMO: the glue that binds. Dev Cell 2006 , 11:596-597.

29. Shen TH, Lin HK, Scaglioni PP, Yung TM, Pandolfi PP: The mechanisms of PML-nuclear body formation. Mol Cell 2006, 24:331-339.

30. Kamitani T, Kito K, Nguyen HP, Wada H, Fukuda-Kamitani T, Yeh ET: Identification of three major sentrinization sites in PML. J Biol Chem 1998 273:26675-26682.

31. Diaz-Griffero F, Li X, Javanbakht H, Song B, Welikala S, Stremlau M, Sodroski $\mathrm{J}$ : Rapid turnover and polyubiquitylation of the retroviral restriction factor TRIM5. Virology 2006, 349:300-315.
32. Sayah DM, Sokolskaja E, Berthoux L, Luban J: Cyclophilin A retrotransposition into TRIM5 explains owl monkey resistance to HIV-1. Nature 2004, 430:569-573.

33. Sastri J, O'Connor C, Danielson CM, McRaven M, Perez P, Diaz-Griffero F, Campbell EM: Identification of residues within the $L 2$ region of rhesus TRIM5alpha that are required for retroviral restriction and cytoplasmic body localization. Virology 2010, 405:259-266.

doi:10.1186/1742-4690-10-10

Cite this article as: Lukic et al:: Role of SUMO-1 and SUMO interacting motifs in rhesus TRIM5a-mediated restriction. Retrovirology 2013 10:10.

\section{Submit your next manuscript to BioMed Central and take full advantage of:}

- Convenient online submission

- Thorough peer review

- No space constraints or color figure charges

- Immediate publication on acceptance

- Inclusion in PubMed, CAS, Scopus and Google Scholar

- Research which is freely available for redistribution
C Biomed Central 